\title{
Use of whole body vibration therapy in individuals with moderate severity of cerebral palsy- a feasibility study
}

\author{
Tamis W. Pin ${ }^{1 *}$, Penelope B. Butler ${ }^{2}$ and Sheila Purves ${ }^{1}$
}

\begin{abstract}
Background: This pilot study was to examine the feasibility and tolerance of whole body vibration therapy (WBVT) for children and adults with moderate severity of cerebral palsy (CP) being graded as levels III or IV on the Gross Motor Function Classification Scale (GMFCS).

Methods: Study participants received the additional WBVT when standing still on the vibration platform for three 3-min bouts of vibration ( $20 \mathrm{~Hz}, 2 \mathrm{~mm}$ amplitude), 4 days per week for 4 weeks. In addition to questions relating to feasibility and participants' opinions, assessment at baseline and completion of the intervention included the Gross Motor Function Measure-66 Item Set (GMFM-66 IS), 2-min walk test (2MWT), Timed Up and Go test (TUG) and Pediatric Evaluation of Disability Inventory (PEDI). Wilcoxon Signed Ranks test was used to compare the results.
\end{abstract}

Results: Fourteen participants (mean age $=25.25$ years SD 3.71; 9 males, 64\%; GMFCS level III $n=13$, 92\%) were recruited and completed the study. The attendance rate was over $90 \%$ with no adverse events. All participants tolerated the protocol which was satisfactorily delivered in a clinical setting.

Conclusions: The present WBVT protocol was feasible, safe and well-tolerated by the participants with moderate severity of $C P$, justifying future studies with larger samples and more rigorous study design.

Trial registration: The present study has been registered under the ClinicalTrials.gov (NCT03375736) and the date of registration commenced on 18 December 2017.

Keywords: Cerebral palsy, Whole body vibration, Functional abilities, Static standing, Balance

\section{Background}

Cerebral palsy $(\mathrm{CP})$ is one of the most common childhood motor disabilities [1]. Although the brain damage is non-progressive, the clinical features resulting from the upper motor neuron lesion cause spasticity, poor motor control, muscle weakness and poor balance. All these features lead to secondary musculoskeletal changes resulting in limb joint contractures and bony deformities which further impact muscle strength and motor function [2]. When compared with their typically developing healthy peers, children and young adults with $\mathrm{CP}$ are thus not able to perform the same amount of exercise to improve their muscle strength and motor function [3].

\footnotetext{
*Correspondence: tamis.pin@polyu.edu.hk

'Department of Rehabilitation Sciences, The Hong Kong Polytechnic

University, Hung Hom, Hong Kong

Full list of author information is available at the end of the article
}

Individuals with $\mathrm{CP}$ are commonly classified into one of five levels according to their physical mobility using the Gross Motor Function Classification System (GMFCS) levels I to $\mathrm{V}^{1}$ [4]. Individuals of GMFCS levels III or IV, whose balance and functional mobility are greatly compromised, have the greatest limitation in performing exercises to improve their muscle strength and motor function [1].

Mechanical vibration has been used as an intervention strategy for neuro-modulation in various neurological diseases $[5,6]$. The vibrations stimulate the alpha-motor neurons of the muscle spindles leading to the tonic vibration reflex $[6,7]$. The reflex then increases voluntary muscle contractions [8]. It has also been shown that the vibrations stimulate peripheral mechanoreceptors, possibly inducing neuroplasticity via somatosensory and motor pathways if applied repeatedly [9]. Furthermore, 
vibration that is provided to the whole body may increase neural drive to the muscles, allowing recruitment of previously inactive motor units [8] with resulting increase in muscle mass and strength.

The vibration can be provided to the whole body or as a focal vibration to a specific muscle or tendon [6]. Previous studies have shown the effectiveness of vibration in reducing spasticity in patients after stroke or spinal cord injuries, in stimulating proprioception and subsequently better motor control during walking in patients with stroke and Parkinson disease, and in enhancing muscle activity during walking in patients with spinal cord injuries [6]. Preliminary findings have shown that vibration may possibly improve the gait pattern for individuals with childhood ataxia [10]. In whole body vibration therapy (WBVT), the user stands in a static position or performs some dynamic movements on a device providing vibrations from a few $\mathrm{Hz}$ to $50 \mathrm{~Hz}(\mathrm{~Hz}$, Hertz represents the number of complete up and down movement cycle per second) [8].

Research on WBVT for both children and adults with CP have appeared to show positive trends in terms of gross motor function, balance, muscle strength and muscle tone [5, 11-14]. There is some suggestion that WBVT improved muscle strength, reduced spasticity, and improved gait parameters and functional activities for children and adolescents with CP [9-13] with no report of any adverse event. However, most studies have included only participants with mild severity of $\mathrm{CP}$. In addition, almost all studies required the study participants to perform simple exercises on the vibration platform $[5,11]$. As far as can be determined, only two published studies have been conducted on children and young adults with more severe CP, i.e. GMFCS level V and these focused only on the bone mineral density of the participants $[15,16]$. Thus, the level of evidence of benefit of WBVT for individuals with moderate severity of $\mathrm{CP}$ (GMFCS levels III and IV) remains unknown, as does the practicality of conducting WBVT for these individuals.

This pilot study aimed to examine the feasibility of a 4-week WBVT programme on children and adults with moderate severity of $\mathrm{CP}$. The specific feasibility objectives were:

i. To investigate the practicality and tolerance of the intervention protocol adapted from the study by Gusso and colleagues [17]. Their study showed positive results on muscle strength and walking ability in young adults with CP of GMFCS levels II and III after 20 weeks but it is noted that this was an uncontrolled study [17].

ii. To investigate if any change in functional abilities and balance resulted from static standing on the vibration platform. iii. To investigate if there were any adverse effects of this intervention for this population group.

iv. To gain information about the study participants' feelings regarding this intervention in terms of comfort and personal opinion.

\section{Methods}

A convenience sample of a total of 10 individuals aged between 6 and 45 years was targeted, recognizing that sample size calculation is not usually required for pilot studies [18]. This broad age range allowed examination of the feasibility of WBVT on a wide spectrum of this population group. The inclusion criteria were participants: (i) with a diagnosis of $\mathrm{CP}$; (ii) with moderate severity of $\mathrm{CP}$, i.e. GMFCS levels III or IV; (iii) able to stand for $3 \mathrm{~min}$ independently or with own hand support on rails; (iv) able to understand simple instructions; and (v) able to tolerate clinical examination. The exclusion criteria were participants: (i) with bone fracture 8 weeks prior to enrolment to the present study, or with acute thrombosis, muscle or tendon inflammation, renal stones, discopathy or arthritis as reported by the participants and/or their parent/guardian; (ii) with metal implants in their spine or lower limbs; (iii) using anabolic agents or growth hormone for at least 1 month, within 3 months prior to enrolment into the present study; and (iv) being pregnant.

The study participants received the WBVT when standing still on the Galileo Med L Plus model 2000 (Novotech Medical GmbH, Germany), wearing their normal clothing and footwear. WBVT was additional to their usual therapy program. The WBVT intervention sessions consisted of three 3-min bouts of vibration of $20 \mathrm{~Hz}$ and a peak-to-peak amplitude of $2 \mathrm{~mm}$ with a 3 -min rest in between based on the study by Gusso and colleagues [17]. The parameters of the vibration were gradually progressed to the desired level in the first seven sessions allowing the study participants to accustom to the intense sensory stimulation from the vibration (Table 2). The intervention took place on 4 days per week for 4 weeks.

All the participants were assessed at baseline and completion of the intervention using the following outcome measures:

i. Gross Motor Function Measure (GMFM-66) Item Set (IS) [19] is a validated and reliable assessment of general gross motor function in various positions including lying, sitting, 4-point kneeling, high kneeling and standing for individuals with CP [20]. The GMFM-66 IS is a condensed version of the GMFM to improve its efficiency of administration in clinical settings [19].

ii. Two-minute walk test (2MWT) to assess submaximal exercise capacity. The distance covered 
in 2 min was measured using a distance-measuring trundle wheel following the published testing protocol [21, 22];

iii. Timed Up and Go (TUG) test to assess balance and functional mobility [23, 24];

iv. A validated Chinese version of the Pediatric Evaluation of Disability Inventory (PEDI) $[25,26]$ to assess functional capacities in the domains of daily activities (73 items), mobility (59 items) and social/cognitive function (65 items); and

v. Visual analogue scale to assess any discomfort associated with the intervention. The scale ranged from 0 (no discomfort) to 10 (extreme discomfort), as reported by the study participants and/or by their carers' proxy. This was accompanied by any subjective comments the participants wished to make.

Ethics approval was granted from the affiliated University of the first and third authors. An informed consent form was signed by the participants over 18 years old, or if not, including all participants under 18 years old, by their parents/guardians. This study was registered under the ClinicalTrials.gov (NCT03375736).

The study results were presented as means and standard deviations. Due to the small sample size, non-parametric Wilcoxon Signed Ranks test was used to compare the results before and after the WBVT. The statistical significance level was set at $p<0.05$.

\section{Results}

Fourteen participants (mean age $=25.25$ years SD 3.71; 9 males, 64\%) were recruited from a special school for students with physical and cognitive impairment $(n=8$, mean age $=14.56$ years, SD 6.78; 5 males, $63 \%)$ and a sheltered workshop cum hostel $(n=6$, mean age $=39.50$ years, SD 3.31; 4 males, 67\%) (Fig. 1). An overwhelming response from the participants and the on-site therapists led to a larger sample size than originally planned. Table 1 shows the characteristics of the study participants. The

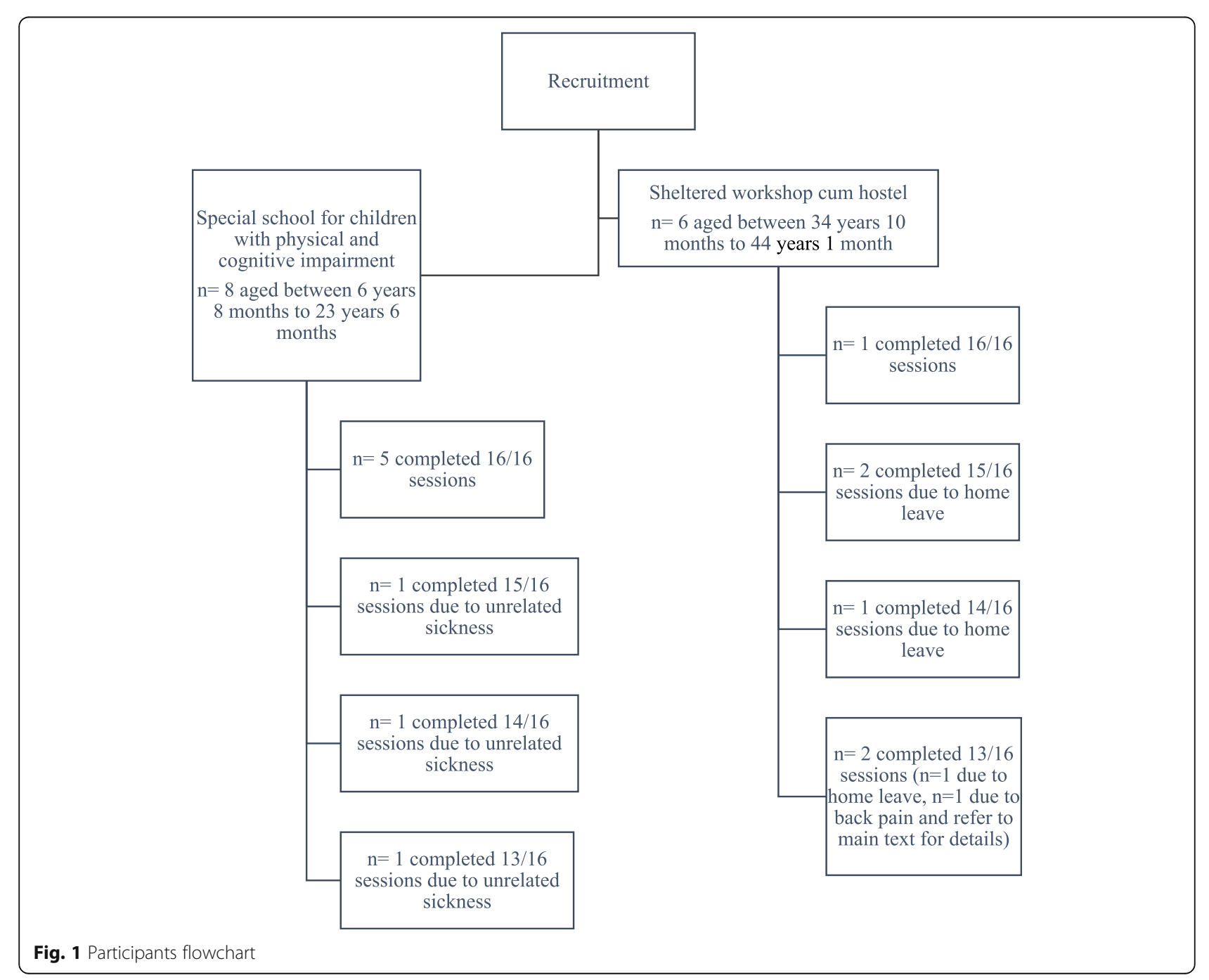


Table 1 Characteristics of study participants

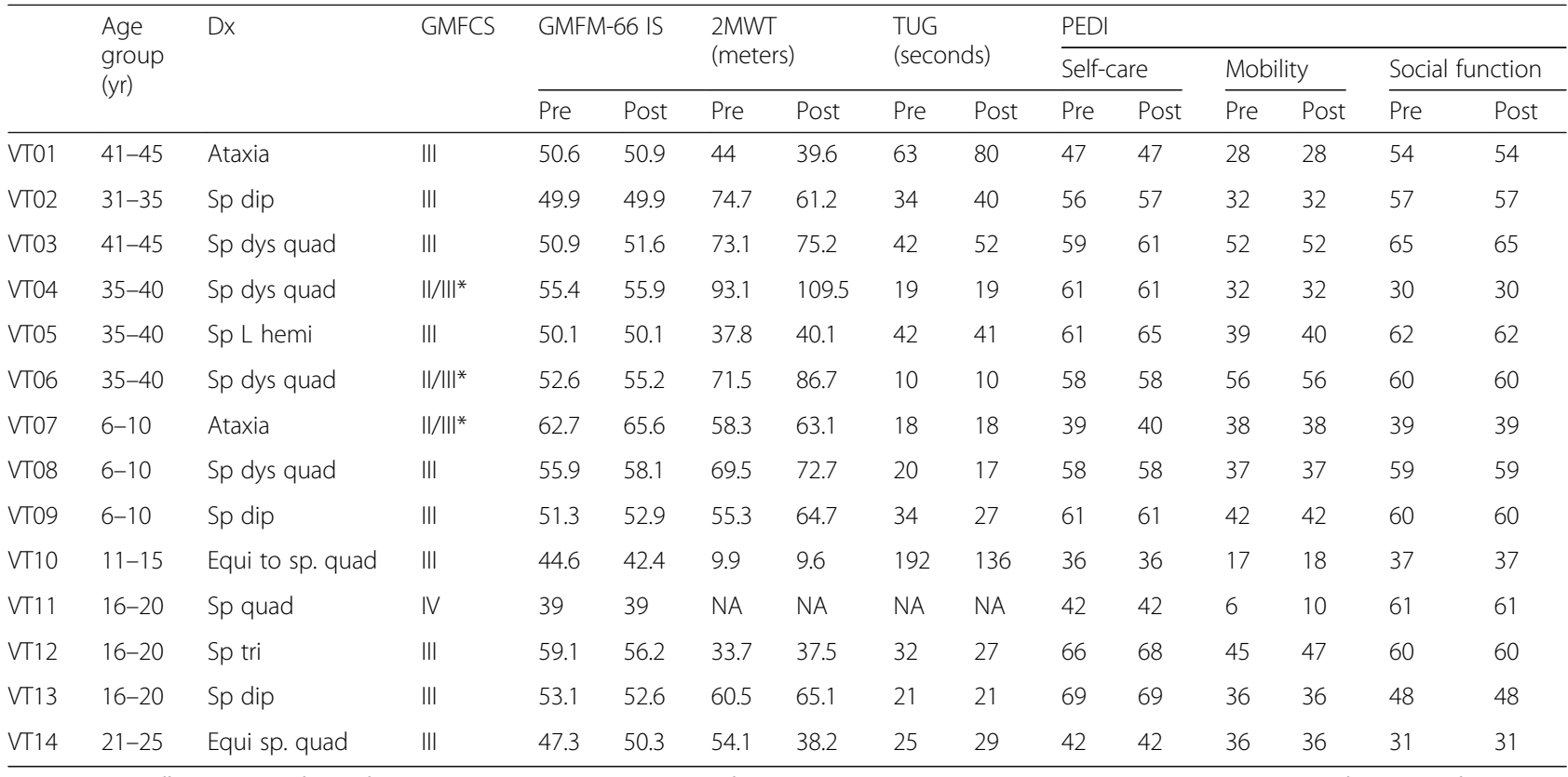

2MWT 2-min walk test, Dip Diplegia, dys Dystonia, Dx Diagnosis, equi Equivalent to, GMFM-66 IS Gross Motor Function Measure 66 Item Set, hemi Hemiplegia, $L$ Left, PEDI Pediatric Evaluation of Disability Inventory, quad Quadriplegia, Sp Spastic, TUG Timed up and go test, tri Triplegia

*These 3 participants had mixed clinical features of GMFCS levels II and III. They all relied on use of a walking aid with supervision (VT7) or wheeled mobility (VT4 and VT6) for long distances in outdoor environments. All were able to walk without a walking aid for short distances in indoor environments but their balance raised safety concerns; hence, VT7 used a walking frame most of the time. Neither VT4 nor VT6 were able to hold any walking aid due to the dystonic features in their upper limbs

NA- data not available as VT11 used a body-weight-support walking frame for therapeutic walking only and required moderate manual assistance

majority of participants functioned at the GMFCS level III $(n=13,92 \%)$.

Two employed physical therapists separately provided the WBVT to all the study participants at their respective recruitment sites. The attendance rate of the study participants was $93.36 \%$ (SD 7.61). The greatest number of missed intervention sessions was three out of 16 sessions for three participants (VT2, VT4 and VT14 in Table 1 and Fig. 1). Six participants completed all 16 sessions. One adult participant (VT4) stumbled when he stepped off the vibration platform after the eighth session. This participant missed the following two sessions of WBVT and requested to re-start the WBVT in the third week. No adverse event was reported for any other participant. All participants coped well with the protocol and the physical therapists had no difficulty in delivering the program.

All the participants were assessed by the first author (TWP) at baseline and after 4 weeks of intervention. The results are presented in Table 1 and graphically in Fig. 2 . The Wilcoxon Signed Rank test showed no significant difference in any of the outcome measures.

\section{Availability of data and materials}

The raw data and data sets used and analyzed in the present study are available from the corresponding author on reasonable request.

\section{Discussion}

This pilot study examined the feasibility of an adapted WBVT protocol for children and adults with moderate severity of $\mathrm{CP}$. The study also investigated any changes in functional abilities and balance.

\section{Feasibility of the intervention protocol}

Most of the previous studies on WBVT required the participants to perform simple exercises, such as mini-squats or lunges, on the vibration platform. This poses a great challenge for individuals with moderate severity of $\mathrm{CP}$ but these are the individuals most vulnerable to poor muscle strength and motor function as a consequence of their greatly compromised mobility [27, 28]. The present study thus investigated the feasibility of static standing on the vibration platform with both knees in slight flexion and with hand support if needed. This protocol (Table 2) was less demanding than the one recommended for children and adolescents with disabilities [5]. Participants tolerated the intervention well and had high completion percentage with the minimal number of reported adverse events: this suggests that the present protocol of the WBVT was feasible, safe and acceptable for the study participants across the given age range. Physical therapists were able to deliver the WBVT without difficulty in a routine clinical environment. 


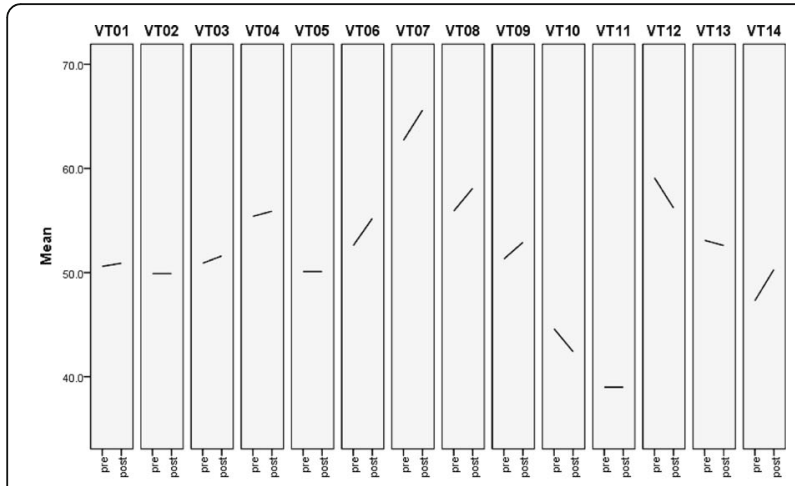
GMFM-66 Item Set total scores
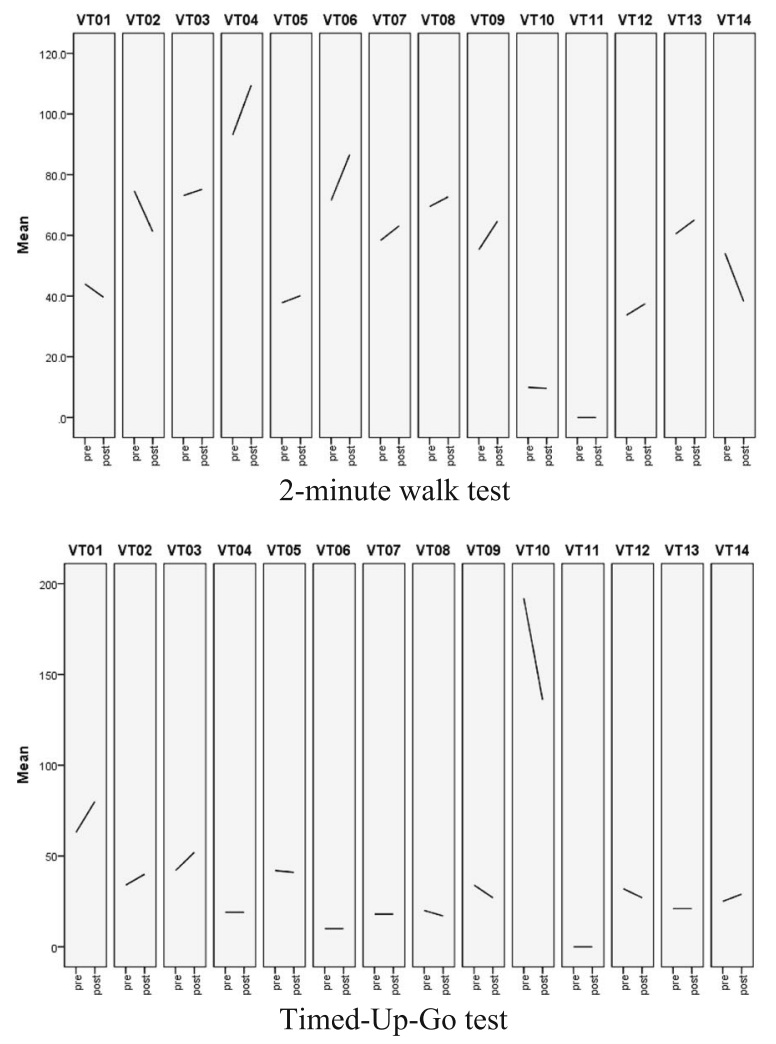

Fig. 2 Graphed study results

\section{Functional abilities and balance}

This feasibility study used the same outcome measures on study participants across a wide age range. Both the 2MWT and TUG have been conducted on both children and adults with the test instructions largely remaining the same [21, 23, 24]. Although the GMFM-66 has been validated only for children with $\mathrm{CP}$, it has also been used as an outcome measure for adults with CP [29-32]. The GMFM-66 IS is a condensed version of the GMFM-66. The PEDI is an outcome measure designed to assess functional performance in daily activities for children aged 9 months to 7.5 years of age but can be used above this age if typical performance has not been achieved [26] as in previous research studies for older individuals [33-35].

In general, small but positive changes were shown in gross motor function (GMFM-66 IS), functional mobility (2MWT), self-care activities (PEDI self-care), and mobility activities (PEDI mobility) (Table 1 and Fig. 2). The improvement in the PEDI self-care activities reached statistical significance and, in mobility activities, a close to statistical significance. This small pilot study is insufficient to verify whether static standing on the WBVT platform confers the same benefit as exercises during WBVT but may indicate a preliminary trend. It was interesting to note that the self-care activities that showed improvement all involved core stability and balance in various positions, such as cleaning or drying the body after a shower, putting toothpaste on the toothbrush and brushing the teeth, or spilling water. Previous studies have shown that muscle strength around the trunk and lower limbs was increased with WBVT, which in turn, improved core stability and reduced dependence on hand support [12, 14, 17, 29]. Participants also found moving in and out of positions easier (PEDI mobility). Participant VT11 showed the largest increase in this score (Table 1 and Fig. 2). He was a young man with spastic quadriplegic $\mathrm{CP}$ and the only participant with GMFCS level IV. He stated that he could roll, get in and out of a chair and bed with more ease and with no increase in carer assistance.

\section{Appendix}

Table $\mathbf{2}$ Intervention protocol of the present study

\begin{tabular}{lllllll}
\hline Session & Vibration 1 & Rest 1 & Vibration 2 & Rest 2 & Vibration 3 \\
\hline 1st & $1 \mathrm{~min} ; 12 \mathrm{~Hz}, 1 \mathrm{~mm}$ & $3 \mathrm{~min}$ & $1 \mathrm{~min} ; 12 \mathrm{~Hz}, 1 \mathrm{~mm}$ & $3 \mathrm{~min}$ & $1 \mathrm{~min} ; 15 \mathrm{~Hz}, 1 \mathrm{~mm}$ & $3 \mathrm{~min}$ \\
2nd & $1 \mathrm{~min} ; 15 \mathrm{~Hz}, 1 \mathrm{~mm}$ & $3 \mathrm{~min}$ & $1 \mathrm{~min} ; 15 \mathrm{~Hz}, 1 \mathrm{~mm}$ & $3 \mathrm{~min}$ & $2 \mathrm{~min} ; 15 \mathrm{~Hz}, 1 \mathrm{~mm}$ \\
3th & $2 \mathrm{~min} ; 15 \mathrm{~Hz}, 1 \mathrm{~mm}$ & $3 \mathrm{~min}$ & $3 \mathrm{~min} ; 15 \mathrm{~Hz}, 1 \mathrm{~mm}$ & $3 \mathrm{~min}$ & $3 \mathrm{~min} ; 15 \mathrm{~Hz}, 1 \mathrm{~mm}$ \\
4th & $2 \mathrm{~min} ; 18 \mathrm{~Hz}, 1 \mathrm{~mm}$ & $3 \mathrm{~min}$ & $2 \mathrm{~min} ; 18 \mathrm{~Hz}, 1 \mathrm{~mm}$ & $3 \mathrm{~min}$ & $2 \mathrm{~min} ; 18 \mathrm{~Hz}, 1 \mathrm{~mm}$ \\
5 th & $3 \mathrm{~min} ; 20 \mathrm{~Hz}, 1 \mathrm{~mm}$ & $3 \mathrm{~min}$ & $3 \mathrm{~min} ; 20 \mathrm{~Hz}, 1 \mathrm{~mm}$ & $3 \mathrm{~min}$ & $3 \mathrm{~min} ; 20 \mathrm{~Hz}, 1 \mathrm{~mm}$ \\
6th & $3 \mathrm{~min} ; 20 \mathrm{~Hz}, 1 \mathrm{~mm}$ & $3 \mathrm{~min}$ & $3 \mathrm{~min} ; 20 \mathrm{~Hz}, 2 \mathrm{~mm}$ & $3 \mathrm{~min}$ & $3 \mathrm{~min} ; 20 \mathrm{~Hz}, 2 \mathrm{~mm}$ & $3 \mathrm{~min}$ \\
$>$ 7th & $3 \mathrm{~min} ; 20 \mathrm{~Hz}, 2 \mathrm{~mm}$ & $3 \mathrm{~min}$ & $3 \mathrm{~min} ; 20 \mathrm{~Hz}, 2 \mathrm{~mm}$ & $3 \mathrm{~min}$ & $3 \mathrm{~min} ; 20 \mathrm{~Hz}, 2 \mathrm{~mm}$ \\
\hline
\end{tabular}

Adapted from the study by Gusso and colleagues (2016) 


\section{Adverse effects}

No participant complained of pain or discomfort during or after the WBVT. We cannot confirm that the stumble experienced by Participant VT4 was linked to the WBVT as he had a history of unexplained falls.

\section{Subjective comments from study participants}

The most frequent comments by the study participants after the WBVT included 'very relaxed' legs (7 out of 14 participants, 50\%) and 'jelly' legs or 'weak' legs (5 out of 14 participants, 36\%). Some vocal adult participants (VT2 and VT12) indicated that their legs felt less stiff. Two participants (VT3 and VT6), both residents in the sheltered workshop cum hostel, commented on the change in walking pattern of Participant VT4 after one of the intervention sessions. VT4 usually walked using a total extensor pattern but used a flat-foot gait pattern after that session. Inclusion of $3 \mathrm{D}$ gait analysis, where practical, could be valuable in future studies or video-recording with analytical tools if kinematic and kinetic measures are unavailable.

\section{Limitations of present study}

This pilot study was with a small sample size and a low level of study design. Repetition of the outcome measures at 4 weeks was a relatively short duration that could have led to some learning effect. However, the primary focus of this study was to ascertain the feasibility of the WBVT program.

The study group were mostly of GMFCS level III and only one participant of GMFCS level IV. Potential participants with more severe $\mathrm{CP}$ were cognitively unable to follow instructions to remain still in the required posture on the vibration platform. This difficulty may be overcome in future studies by providing WBVT on a standing tilt-table with an installed vibration platform.

Overall, the present preliminary findings justify future studies with more rigorous study designs and larger samples that include more participants with greater severity of CP (GMFCS level IV) before the present WBVT protocol is recommended for use in clinical settings.

\section{Conclusions}

The present pilot study has shown that the proposed WBVT treatment protocol is feasible, safe and acceptable for children, adolescents and adults with moderate severity of $\mathrm{CP}$ and is practical in a routine clinical setting. There were small trends towards improvement in functional abilities and balance with this static standing protocol. These findings justify use of the same protocol on a larger sample size of this population group using a more rigorous study design.

\section{Endnotes}

${ }^{1}$ In general, individuals of levels I and II ambulate unaided and are able to perform more advanced motor skills such as running and jumping with limitations. Individuals of level III mobilise with hand-held mobility devices such as crutches or walking frames. Individuals of levels IV have very limited functional mobility and are mostly limited to an indoor environment. Individuals with level $\mathrm{V}$ are non-ambulatory or totally rely on wheeled mobility aids in all environment settings.

\begin{abstract}
Abbreviations
2MWT: 2-min walk test; CP: Cerebral palsy; GMFCS: Gross Motor Function Classification Scale; GMFM-66 IS: Gross Motor Function Measure 66 Item Set; PEDI: Pediatric Evaluation of Disability Inventory; TUG: Timed-up-go test; WBVT: Whole body vibration therapy
\end{abstract}

\section{Acknowledgements}

The authors would thank Miss Joyce HL Choi for her assistance in data collection and management, all the study participants and the staff at the SAHK Erik Kvan Workshop and Hostel and the Hong Kong Red Cross Society John F Kennedy Centre, and Ms. Suzanne Cheung and Ms. Tina Chan for their provision of on-site intervention to the study participants.

\section{Funding}

The present study was fully financed by The Hong Kong Polytechnic University internal research fund granted to Dr. Tamis W Pin. The funding body is not involved in the study design, collection, analyses, and interpretation of the data; in the writing of the manuscript, and in the decision to submit the manuscript for publication.

\section{Availability of data and materials}

The datasets used and/or analysed during the current study are available from the corresponding author on reasonable request.

\section{Authors' contributions}

TWP designed the study, collected, analysed and interpreted the data, wrote the manuscript and was fund-holder of this study. PBB and SP designed the study, analysed, interpreted the data, and significantly contributed to writing the manuscript. All authors read and approved the final manuscript.

\section{Ethics approval and consent to participate}

This research study was conducted in accordance with the Declaration of Helsinki. Ethics approvals were granted from the Departmental Research Committee, Department of Rehabilitation Sciences, The Hong Kong Polytechnic University (HSEARS20171123001). Participants over 18 years of age and parents of all participants under 18 years of age signed an informed consent before data collection.

Consent for publication

Not applicable.

\section{Competing interests}

The authors declare that they have no competing interests.

\section{Publisher's Note}

Springer Nature remains neutral with regard to jurisdictional claims in published maps and institutional affiliations.

\section{Author details}

'Department of Rehabilitation Sciences, The Hong Kong Polytechnic University, Hung Hom, Hong Kong. ${ }^{2}$ Manchester Metropolitan University, Manchester, UK. 
Received: 11 September 2018 Accepted: 15 April 2019

Published online: 01 May 2019

\section{References}

1. Himmelmann K, Beckung E, Hagberg G, Uvebrant P. Gross and fine motor function and accompanying impairments in cerebral palsy. Dev Med Child Neurol. 2006;48(6):417-23.

2. Peacock WJ. The neural contral of movement. In: Gage JR, Schwartz MH, Koop SE, Novacheck TF, editors. The Idenification of treatment of gait problems in cerebral palsy. 2nd edn. London: Mac Keith Press; 2009. p. 3-20.

3. van den Berg-Emons HJG, Saris WHM, de Barbanson DC, Westerterp KR, Huson A, van Baak MA. Daily physical activity of schoolchildren with spastic diplegia and of healthy control subjects. J Pediatr. 1995;127(4):578-84.

4. Rosenbaum PL, Palisano RJ, Bartlett DJ, Galuppi BE, Russell DJ. Development of the gross motor function classification system for cerebral palsy. Dev Med Child Neurol. 2008;50(4):249-53.

5. Matute-Llorente A, Gonzalez-Aguero A, Gomez-Cabello A, Vicente-Rodriguez G, Casajus Mallen JA. Effect of whole-body vibration therapy on healthrelated physical fitness in children and adolescents with disabilities: a systematic review. J Adolesc Health. 2014;54(4):385-96.

6. Murillo N, Valls-Sole J, Vidal J, Opisso E, Medina J, Kumru H. Focal vibration in neurorehabilitation. Eur J Phy Rehabil Med. 2014;50(2):231-42.

7. Rehn B, Lidstrom J, Skoglund J, Lindstrom B. Effects on leg muscular performance from whole-body vibration exercise: a systematic review. Scand J Med Sci Sport. 2007;17(1):2-11.

8. Jordan MJ, Norris SR, Smith DJ, Herzog W. Vibration training: an overview of the area, training consequences, and future considerations. J Strength Cond Res. 2005;19(2):459-66.

9. Lopez S, Bini F, Del Percio C, Marinozzi F, Celletti C, Suppa A, Ferri R, Staltari E, Camerota F, Babiloni C. Electroencephalographic sensorimotor rhythms are modulated in the acute phase following focal vibration in healthy subjects. Neuroscience. 2017;352:236-48.

10. Schirinzi T, Romano A, Favetta M, Sancesario A, Burattini R, Summa S, Della Bella G, Castelli E, Bertini E, Petrarca M, et al. Non-invasive focal mechanical vibrations delivered by wearable devices: an open-label pilot study in childhood Ataxia. Front Neuro. 2018;9:849.

11. Saquetto M, Carvalho V, Silva C, Conceicao C, Gomes-Neto M. The effects of whole body vibration on mobility and balance in children with cerebral palsy: a systematic review with meta-analysis. J Musculoskelet Neuronal Interact. 2015;15(2):137-44

12. Cheng HY, Yu YC, Wong AM, Tsai YS, Ju YY. Effects of an eight-week whole body vibration on lower extremity muscle tone and function in children with cerebral palsy. Res Dev Disabil. 2015;38:256-61.

13. Ko M-S, Doo J-H, Kim J-S, Jeon H-S. Effect of whole body vibration training on gait function and activities of daily living in children with cerebral palsy. Int J Ther Rehabil. 2015;22(7):321-8.

14. Unger M, Jelsma J, Stark C. Effect of a trunk-targeted intervention using vibration on posture and gait in children with spastic type cerebral palsy: a randomized control trial. Dev Neurorehabil. 2013;16(2):79-88.

15. Dalén Y, Sääf M, Nyrén S, Mattsson E, Haglund-Åkerlind Y, Klefbeck B. Observations of four children with severe cerebral palsy using a novel dynamic platform. A case report. Adv Physiother. 2012;14(3):132-9.

16. Kilebrant $\mathrm{S}$, Braathen $\mathrm{G}$, Emilsson $\mathrm{R}$, Glansén U, Söderpalm A-C, Zetterlund B, Westerberg B, Magnusson P, Swolin-Eide D. Whole-body vibration therapy in children with severe motor disabilities. J Rehabil Med. 2015:47(3):223-8.

17. Gusso S, Munns CF, Colle P, Derraik JG, Biggs JB, Cutfield WS, Hofman PL. Effects of whole-body vibration training on physical function, bone and muscle mass in adolescents and young adults with cerebral palsy. Sci. 2016;6:22518.

18. Thabane L, Ma J, Chu R, Cheng J, Ismaila A, Rios LP, Robson R, Thabane M, Giangregorio L, Goldsmith CH. A tutorial on pilot studies: the what, why and how. BMC Med Res Methodol. 2010;10(1):1.

19. Russell D, Avery L, Walter S, Hanna S, Bartlett D, Rosenbaum P, Palisano R, Gorter JW. Development and validation of item sets for the GMFM- 66 to improve the efficiency of administration in children with cerebral palsy. Dev Med Child Neurol. 2009:51(Supplement(2)):89-90.

20. Russell D, Rosenbaum P, Wright M, Avery LM. Gross motor function measure (GMFM-66 \& GMFM-88) Use's manual. 2nd ed. Ontario: Mac Keith Press; 2013.

21. Pin TW. Psychometric properties of 2-minute walk test: a systematic review. Arch Phys Med Rehabil. 2014;95:1759-75.
22. Pin TW, Choi HL. Reliability, validity, and norms of the 2-min walk test in children with and without neuromuscular disorders aged 6-12. Disabil Rehabil. 2018:40(11):1266-72.

23. Podsiadlo D, Richardson S. The timed "up \& go": a test of basic functional mobility for frail elderly persons. J Am Geriatr Soc. 1991;39(2):142-8.

24. Williams EN, Carroll SG, Reddihough DS, Phillips BA, Galea MP. Investigation of the timed 'up \& go' test in children. Dev Med Child Neurol. 2005;47(8):518-24.

25. Haley SM, Coster WJ, Ludlow LH, Haltiwanger JT, Andrellos PJ. Pediatric evaluative disability index. Boston: PsyCorp; 1992.

26. Chen K-L, Hsieh C-L, Ching-Fan Sheu C-F, Hu F-C, Tseng M-H. Reliability and validity of a Chinese version of the pediatric evaluation of disability inventory in children with cerebral palsy. J Rehabil Med. 2009;41:273-8.

27. Apkon SD. Osteoporosis in children who have disabilities. Phys Med Rehabil Clin N Am. 2002;13(4):839-55.

28. Sheridan KJ. Osteoporosis in adults with cerebral palsy. Dev Med Child Neurol. 2009;51(Suppl 4):38-51.

29. Ahlborg L, Andersson C, Julin P. Whole-body vibration training compared with resistance training: effect on spasticity, muscle strength and motor performance in adults with cerebral palsy. J Rehabil Med. 2006;38(5):302-8.

30. Andersson C, Grooten W, Hellsten M, Kaping K, Mattsson E. Adults with cerebral palsy: walking ability after progressive strength training. Dev Med Child Neurol. 2003:45(4):220-8

31. Maltais DB, Robitaille NM, Dumas F, Boucher N, Richards CL. Measuring steadystate oxygen uptake during the 6-min walk test in adults with cerebral palsy: feasibility and construct validity. Int J Rehabil Res. 2012;35(2):181-3.

32. Reynolds MR, Ray WZ, Strom RG, Blackburn SL, Lee A, Park TS. Clinical outcomes after selective dorsal rhizotomy in an adult population. World Neurosurg. 2011;75(1):138-44.

33. Odman PE, Oberg BE. Effectiveness and expectations of intensive training: a comparison between child and youth rehabilitation and conductive education. Disabil Rehabil. 2006:28(9):561-70.

34. Ohata K, Tsuboyama T, Haruta T, Ichihashi N, Kato T, Nakamura T. Relation between muscle thickness, spasticity, and activity limitations in children and adolescents with cerebral palsy. Dev Med Child Neurol. 2008;50(2):152-6.

35. van Empelen R, Jennekens-Schinkel A, Gorter JW, Volman MJ, van Nieuwenhuizen $\mathrm{O}$, Helders PJ, Dutch collaborative epilepsy surgery P. Epilepsy surgery does not harm motor performance of children and adolescents. Brain. 2005;128(Pt 7):1536-45.

\section{Ready to submit your research? Choose BMC and benefit from:}

- fast, convenient online submission

- thorough peer review by experienced researchers in your field

- rapid publication on acceptance

- support for research data, including large and complex data types

- gold Open Access which fosters wider collaboration and increased citations

- maximum visibility for your research: over $100 \mathrm{M}$ website views per year

At BMC, research is always in progress.

Learn more biomedcentral.com/submissions 